\title{
DATA ASSIMILATION OF SATELLITE IMAGES WITHIN AN OCEANOGRAPHIC CIRCULATION MODEL
}

\author{
E. Huot ${ }^{\dagger}$ \\ T. Isambert ${ }^{\ddagger}$ \\ I. Herlin \\ J.-P. Berroir ${ }^{\ddagger}$ \\ G. Korotaev ${ }^{\star}$ \\ ${ }^{\dagger}$ Université de Versailles - Saint-Quentin - CETP \\ $\ddagger$ Institut National de Recherche en Informatique et Automatique - équipe Clime \\ ${ }^{\star}$ National Academy of Sciences of Ukraine - MHI.
}

\begin{abstract}
We tackle the problem of coupling a geophysical simulation model with data coming from image processing. It needs to define the image observation space and to design an operator to transform results from the image space to the model space. In this study, we use a shallow-water oceanographic circulation model developed at MHI. We propose a processing chain first based on an image processing step relying on a dedicated motion estimation operator, and then a data assimilation step of the estimated velocity. We illustrate the method on different results without and with assimilation.
\end{abstract}

\section{OBJECTIVES}

In the framework of numerical forecasting for the evolution of geophysical fluid, we are interested in the assimilation of data coming from images. In order to forecast the behavior of geophysical fluids we need: a forecast model to describe the evolution of a state variable (generally it is a non-linear PDE system) and observations spatially and temporally distributed. Data assimilation provides a mathematical solution to combine data and models. During last decades model quality increased significantly. But forecast quality is not directly linked to model quality. To increase forecast quality it is also necessary to increase the amount and the quality of observations. Therefore, images - particularly images coming from spatial remote sensing - provide a huge amount of information. Using images in a data assimilation framework raises several difficulties:

1. First it is necessary to define which image space is relevant according to model specialists.

2. Then, it is necessary to construct an image operator dedicated to the problematic and coherent to the physical behavior. Moreover it is necessary to define a set of norm in order to quantify the influence of image information along the assimilation process

3. And finally, it is necessary to construct an operator to compare image space and model space.

In the study presented in this paper, we are interested in oceanographic circulation forecasting. Oceanographic circulation is ruled by fluid mechanic. Most of oceanographic circulation models are heavy 3D models based on primitive equations [1]. They correspond to an approximation of Navier-Stokes equation associated to a nonlinear state equation coupling salinity, temperature and 3D velocity. Nevertheless, it exists simplified models based on shallow-water approximation $[2,3]$. They rely on a so called 1.5 layer representation of the ocean: the sea surface is represented by a mixed layer interfaced to the atmosphere and a deeper layer. Equation ruling the circulation are then:

$$
\left\{\begin{array}{l}
\frac{d u}{d t}-f v=g^{\prime} \frac{\partial h}{\partial x}+\frac{\tau^{(x)}}{\rho_{0} h}+A_{h} \Delta u \\
\frac{d v}{d t}+f u=g^{\prime} \frac{\partial h}{\partial y}+\frac{\tau^{(y)}}{\rho_{0} h}+A_{h} \Delta v \\
\frac{\partial h}{\partial t}+\frac{\partial(u h)}{\partial x}+\frac{\partial(v h)}{\partial y}=0 .
\end{array}\right.
$$

where: $\mathbf{v}$ and $h$ are the state variables, $\mathbf{v}=(u, v)$ is the speed velocity of the mixed layer, $h$ is the thickness of the mixed layer; and $\vec{\tau}=\left(\tau^{(x)}, \tau^{(y)}\right)$ corresponds to the wind stress, $A_{h}$ is the horizontal diffusivity of the mixed layer, and $g^{\prime}=g\left(\rho_{0}-\rho_{1}\right) / \rho_{0}$ is called the reduced gravity with $\rho_{0}$ corresponding to the reference density and $\rho_{1}$ to the average density of the mixed layer. The model used in this study is based on these shallow-water equations and is specially calibrated for the Black Sea. It has been developed in Ukraine in the Dynamics of Oceanic Processes department of the Marine Hydrophysical Institute [4].

There are many oceanographic sources of observation, mainly coming from space remote sensing. Images provided by optical sensors, such as Sea Surface Temperature (SST), present a high temporal coherence. Moreover, temperature is a circulation tracer. Then, the image space we have chosen corresponds to apparent motion velocity fields. The major advantage of using such an image space is that velocity is a variable state of oceanographic circulation model (1). Hence, the operator to compare image space and model space is reduced to a projection. The SST is provided by NOAA-AVHRR satellite sensors. Their spatial resolution is $1.1 \mathrm{~km}^{2}$ and temporal frequency of the satellite is at best one day. But, we usually have several acquisitions for the same day coming from different satellites using the same sensor. Figure 1 displays a SST image acquired in July 14th, 1998. These images present several artefacts such as:

(1) problems due to the geometry acquisition: unavailable information appearing as black and white spots,

(2) clouds: colder than the sea surface they appear darker on images,

(3) problems due to sensor saturation: highly bright zones,

(4) large spatial variation of the local temperature average,

(5) high temporal variation of the global temperature average between acquisitions: due to solar exposition at the acquisition time and to different sensors calibration.

We need to construct a dedicated operator to estimate circulation velocity. The problem of motion estimation from image sequences 


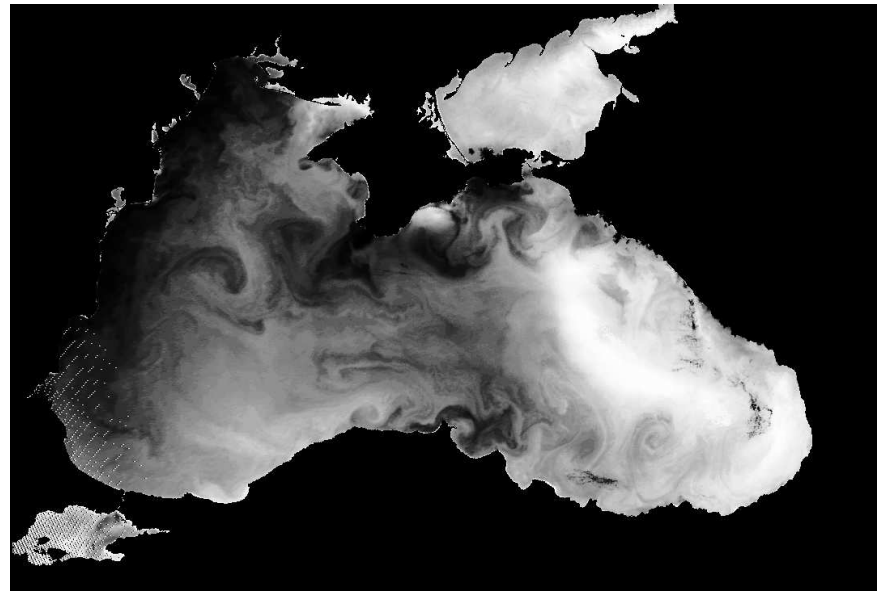

Fig. 1. SST image of the Black Sea given by NOAA/AVHRR.

has been extensively studied $[5,6,7,8,9,10,11,12,13]$. The classical computer vision approach relies on a dense estimation of the displacement velocity based on the so-called conservation of gray level-value assumption (also called optical flow constraint): given a pixel $(x(t), y(t))$ on an image $I$ at time $t$, we have:

$$
\frac{d I}{d t}(x, y, t)=\nabla I \cdot \mathbf{w}+\frac{\partial I}{\partial t}=0,
$$

where $\nabla I$ is the spatial gradient, $\mathbf{w}$ is the displacement between two consecutive images, and - denotes the scalar product. Equation (2) expresses the pixel's luminance conservation over time: moving points keep a constant brightness during their motion. This equation has two unknowns: $\mathbf{w}=(u, v)$ and cannot be directly solved. One classical approach is to add an additional constraint, for example a $L_{2}$ regularity for $\mathbf{w}$, and to express it within an energy minimization framework to obtain an estimation of $\mathbf{w}$.

Due to their special artefacts, oceanographic satellite images must be preprocessed in order to use such kind of dense motion estimation approaches. The section 2 presents the whole processing chain. Image preprocessing is presented in subsection 2.1. Subsection 2.2 defines a motion estimator dedicated to the oceanographic application, $i e$ : define conservation constraint and regularity equation that we have to use and how do we solve the minimization problem, in order to better adapt the operator to the specific context of acquisition process and observed phenomena. And subsection 2.3 presents the assimilation of estimated velocities into the circulation model. The section 3 presents different results with and without assimilation of estimated velocities, and compare them to results obtained using assimilation of other quantities. Finally section 4 gives conclusions and several perspectives to this work.

\section{PROCESSING CHAIN}

\subsection{Image preprocessing}

As we have seen in section 1, SST images coming from NOAA/AVHRR present some artefacts able to compromise estimation of apparent motion with a dense estimator. We need to propose an adapted processing for each artefact.

Artefacts (1) no acquisition, (2) clouds, and (3) sensor saturation have to be masked. The mask is obtain by thresholding the original image:

1. pixels not corresponding to an effective acquisition have either a temperature equal to $0^{\circ} \mathrm{C}$ or greater than $25^{\circ} \mathrm{C}$;

2. clouds have a lower temperature than the ocean surface, pixels with a temperature lower than $10^{\circ} \mathrm{C}$ are considered to be clouds;

3. temperatures greater than $23^{\circ} \mathrm{C}$ are considered to be in the saturation zone of the sensor.

Finally we keep in consideration only sea surface temperatures between $10^{\circ} \mathrm{C}$ and $23^{\circ} \mathrm{C}$, other pixels are masked and not taken into account into the estimation process.

The correction of artefacts (4) and (5) corresponding to the high spatio-temporal variations of the temperature is crucial. It is directly linked to the evaluation of derivatives, mandatory step to solve dense motion estimation equations. To correct the high temporal variation Vigan [14] proposed to apply a low-pass filter to $\partial T / \partial t$ in the Fourier domain. These kind of approach present the drawback to be difficult to tune because it assume a periodical aspect difficult to quantify. Moreover it begins difficult to deal with masks in the frequency domain. We have chosen to correct artefacts (4) in the spatial domain and then to correct artefacts (5): we consider large scale space phenomena to be extended at about $150 \mathrm{~km}^{2}$, we compute average temperature on $150 \times 150 \mathrm{~km}$ windows and subtract this average to the temperature of each pixel, it corrects the large spatial variation; once this correction done, we compute the global average for each image and deduce the mean bias for each couple of images, it corrects the high temporal variation.

\subsection{Estimation of circulation velocities}

In the context of fluid motion on oceanographic images, we are now able to keep the dense estimation framework and its energy minimization, but we need to further analyze the two components of the energy function to be minimized: conservation equation and regularity constraint, with respect the physical underlying process.

In order to investigate which conservation equation and regularity constraint are best adapted, we have used synthetic data coming from the 3D simulation model OPA [15] developed in the LOCEAN laboratory. We have shown in [16] that the best conservation equation to estimate the motion is:

$$
\nabla T \cdot \mathbf{w}+\frac{\partial T}{\partial t}=0
$$

$i e$ : the gray level value conservation (2) applied to temperature. This is coherent with the physical equation of the horizontal temperature transport, the horizontal diffusivity is negligible compared to the frequency of acquisition. But we have also seen in [16] that equation (3) is not respected everywhere. Actually, we have shown that there is two cases where the conservation equation isn't verified: on specific oceanographic structures called filaments and when image information is not reliable (in the case of low spatial or temporal derivatives). We have designed an image based criteria to select points where we expect a rather good estimation according to the conservation assumption. We obtain a selection of reliable points discarding both filaments (using a presegmentation process) and low information pixels (using thresholds on the motion index $T_{t} / \nabla T$ ).

In the same way, we have to choose which regularization criteria is the best adapted to the oceanographic context. By using synthetic data, we have shown that spatial regularity of the velocity norm $L_{2}$ 
[10] or $L_{1}$ [9] are not well adapted to fluid motion. The best criteria is based on the div/curl operators able to make the difference between irrotational and solenoidal components of the motion field:

$$
\min \int_{i m g} \alpha\|\nabla \operatorname{div} \mathbf{w}\|^{2}+\beta\|\nabla \operatorname{curl} \mathbf{w}\|^{2}
$$

where $\alpha$ and $\beta$ are coefficients used to weight respectively the irrotational and solenoidal components. They are calibrated for this study in order to penalize the divergence according to uncompressible fluid properties.

In order to take into account the selection of reliable points and to avoid to handle 4th order PDE, the method we use to solve the minimization problem is based on spline vector field interpolation $[17,18]$. The method tends to reconstruct a dense motion field from a set of observation corresponding to projection of motion vectors onto the image gradient. The vector field $\mathbf{w}$ is constrained to comply with the conservation equation on the reliable points $r_{i}$ and to verify the div/curl regularity on the whole image:

$$
\left\{\begin{array}{l}
\nabla T \cdot \mathbf{w}\left(r_{i}\right)=-\frac{\partial T}{\partial t}\left(r_{i}\right) i \in\{1 \ldots N\} . \\
\min \int_{i m g} \alpha\|\nabla \operatorname{divw}\|^{2}+\beta\|\nabla \operatorname{curlw}\|^{2}
\end{array}\right.
$$

The above system admits an unique solution, which can be determined explicitly.

\subsection{Assimilation of estimated velocities}

Data assimilation is a global mathematical framework for combining data and mathematical model equations, in order to restore as best as possible the state variables of the model. Its principle consists of finding the analyze $a$ of the state variable $X$ according to observations $X_{o b s}$. Sequential data assimilation approaches, like Kalman based methods compute the analyze as:

$$
a=\mathbf{K}\left[X_{o b s}-\mathbf{H} X\right]+X
$$

where $\mathbf{H}$ stands for the observation operator and $\mathbf{K}$ is the Kalman matrix. In this study, we use a simplification of Kalman approaches, a nudging method, where the analyze is given by:

$$
a=\lambda\left[X_{o b s}-\mathbf{H} X\right]+X,
$$

the Kalman matrix is replaced by a constant coefficient $\lambda$ a priori evaluated and called the nudging term.

Using this nudging approach we can introduce the estimated velocity $\mathbf{w}=\left(u_{o b s}, v_{o b s}\right)$ into the system (1) such as:

$$
\left\{\begin{array}{l}
\frac{d u}{d t} \quad-f v=g^{\prime} \frac{\partial h}{\partial x}+\frac{\tau^{(x)}}{\rho_{0} h}+A_{h} \Delta u \\
+\lambda\left[\frac{u_{o b s}}{\sqrt{u_{o b s}^{2}+v_{o b s}^{2}}}-\frac{u}{\sqrt{u^{2}+v^{2}}}\right] \times\left(\sqrt{u^{2}+v^{2}}\right) \\
\frac{d v}{d t} \quad+f u=g^{\prime} \frac{\partial h}{\partial y}+\frac{\tau^{(y)}}{\rho_{0} h}+A_{h} \Delta v \\
+\lambda\left[\frac{v_{o b s}}{\sqrt{u_{o b s}^{2}+v_{o b s}^{2}}}-\frac{v}{\sqrt{u^{2}+v^{2}}}\right] \times\left(\sqrt{u^{2}+v^{2}}\right) \\
\frac{\partial h}{\partial t}+\frac{\partial(u h)}{\partial x}+\frac{\partial(v h)}{\partial y}=0 .
\end{array}\right.
$$

Table 1. Comparison of velocity fields obtain with and without assimilation.

\section{CONCLUSION AND PERSPECTIVES}

In this study we have tackled the problem of data assimilation of information coming from images into a simulation model. We have designed an image operator dedicated to SST images and oceanographic application in order to estimate dense velocity fields. The oceanographic circulation model used is a 2D shallow-water model. We have considered that the estimated apparent velocity was comparable to the shallow-water velocity in order to use estimations as velocity observations. The assimilation method used is based on a nudging approach. In this framework we have demonstrate the potentialities of data assimilation methods.

We have two levels of perspectives for this work. The first level is the direct continuation of this study: we can enhance both the velocity estimator (by adding new a priori information) and the assimilation method (better estimation of the nudging term or use of Kalman approaches). The second level is a general perspective for 

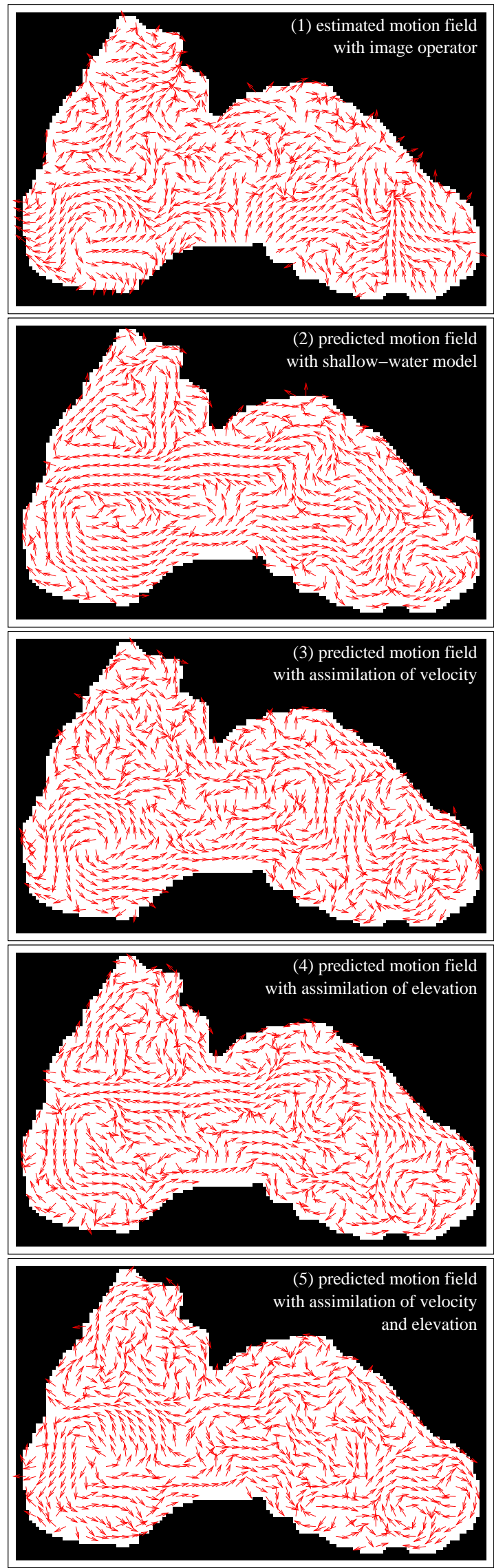

Fig. 2. Different results with and without assimilation. image data assimilation: we should assimilate other kind of structures coming from oceanographic images, such as eddies, fronts, filaments, etc.

\section{REFERENCES}

[1] R. Stewart, Introduction to Physical Oceanography, Department of Oceanography, Texas A\&M University, 2002.

[2] E. Blayo, "Modélisation numérique et assimilation de données en océanographie - Habilitation à Diriger des Recherches," 2002.

[3] B. de Saint-Venant, "Théorie du movement non-permanent des eaux," Compte-Rendu de l'Académie des Sciences, Paris, 1871.

[4] G. Korotaev, T. Oguz, A. Nikiforov, and C. Koblinsky, "Seasonal, interanual, and mesoscale variability of the Black Sea upper layer circulation derived from altimeter data," Journal of Geophysical Research, vol. 108, no. C4, 3122, 2003.

[5] L. Alvarez, J. Weickert, and J. Sanchez, "Reliable estimation of dense optical flow fields with large displacements," IJCV, vol. 39, no. 1, pp. 41-56, August 2000.

[6] J. Barron, D. Fleet, and S. Beauchemin, "Performance of optical flow techniques," International Journal of Computer Vision, vol. 12, no. 1, pp. 43-77, 1994.

[7] S.S. Beauchemin and J.L. Barron, "The computation of optical flow,” Surveys, vol. 27, no. 3, pp. 433-467, September 1995.

[8] D. Béréziat, I. Herlin, and L. Younes, "A generalized optical flow constraint and its physical interpretation," in Proceedings of CVPR'2000, 2000.

[9] I. Cohen and I. Herlin, "Optical flow and phase portrait methods for environmental satellite image sequences," in ECCV, 1996, vol. 2, p. 141.

[10] B.K.P. Horn and B.G. Schunk, "Determining optical flow," Artificial Intelligence, vol. Vol 17, pp. 185-203, 1981.

[11] A. Mitiche and P. Bouthemy, "Computation and analysis of image motion: A synopsis of current problems and methods," International Journal of Computer Vision, vol. Vol 19, no. 1, pp. 29-55, 1996.

[12] H.H. Nagel, "Displacement vectors derived from second-order intensity variations in image sequences," Computer Graphics Image Processing, vol. 21, pp. 85-117, 1983.

[13] R.P. Wildes and M.J. Amabile, "Physically based fluid flow recovery from image sequences," in CVPR97, 1997, pp. 969975.

[14] X. Vigan, C. Provost, R. Bleck, and P. Courtier, "Sea surface velocities from Sea Surface Temperature image sequences," Journal of Geophysical Research, August 2000.

[15] G. Madec, M. Imbard, and C. Lévy, OPA 8.1 Ocean General Circulation Model Reference Manual, Institut Pierre Simon Laplace, Paris, 1999, Notes scientifiques du pôle modélisation.

[16] I. Herlin, F. X. Le Dimet, E. Huot, and J. P. Berroir, Coupling models and data: which possibilities for remotely-sensed images?, chapter e-Environement: progress and challenges, pp. 365-383, Instituto Politécnico Nacional, México, 2004.

[17] L. Amodei, "A vector spline approximation," Journal of approximation theory, vol. 67, pp. 51-79, 1991.

[18] D. Suter, "Motion estimation and vector splines," in CVPR94, 1994. 